\title{
Outcomes of hepatitis C virus treatment with ledipas- vir/sofosbuvir in Mongolian population: Successes and challenges facing scale-up of care
}

\author{
Seong Hee Kang ${ }^{1,2}$ and Moon Young Kim ${ }^{1,2}$ \\ 'Department of Internal Medicine, ${ }^{2}$ Regenerative Medicine Research Center, Yonsei University Wonju College of Medicine, Wonju, Korea
}

Keywords: Hepatitis C, Chronic; Lediapsvir; Sofosbuvir

\section{See Article on Page 125}

The global incidence of chronic hepatitis C virus (HCV) infection is estimated to be approximately 71 million, ${ }^{1}$ and it remains a major cause of chronic liver disease worldwide. ${ }^{2}$ The introduction of oral direct-acting antivirals (DAAs) therapy since 2014 has revolutionized chronic hepatitis $\mathrm{C}(\mathrm{CHC})$ treatment. The currently available DAAs allow the achievement of a sustained viral response (SVR) rate of over $95 \%$ besides demonstrating a satisfactory tolerability. Several sofosbuvir (SOF)-based regimens have demonstrated excellent efficacy and safety for CHC. Among them, ledipasvir (LDV)/SOF has been approved for the treatment of genotypes $1,4,5$, and 6 in the USA and Europe. ${ }^{3,4}$ Its approval provided the first "one pill a day" regimen for 12 weeks as treatment for CHC. As proof-of-concept studies revealed the efficacy and safety of LDV/SOF for genotype 2 HCV infection, LDV/SOF was further evaluated in phase III trials, which led to its label extension to genotype 2 in Canada, followed by Japan, Taiwan, and Korea. ${ }^{5}$
In this issue of Clinical and Molecular Hepatology, Baatarkhuu et al. ${ }^{6}$ presented a study demonstrating the efficacy and safety of LDV/SOF in 5,028 Mongolian patients infected with genotype $1 \mathrm{HCV}$ who received LDV/SOF or LDV/SOF plus ribavirin for 12 weeks. Different SVR12 rates were observed in patients with genotype $1 \mathrm{a}(69.6 \%$, 16 of 23 patients) and genotype $1 \mathrm{~b}(99.7 \%$, 4,992 of 5,005 patients). Among genotype 1b patients, SVR12 rates $>95 \%$ were achieved even in subgroups, such as treatmentexperienced or cirrhosis patients. However, the SVR rate in genotype la was considerably lower than that in previous studies. The author explained that this may be attributed to the presence of NS5A resistance-associated substitutions (RASs) and the small number of enrolled patients with genotype $1 a(n=23)$. According to analysis from 35 clinical trials, LDV-specific RASs were present in $8-13 \%$ of genotype $1 \mathrm{a}$ and $16-18 \%$ of genotype $1 \mathrm{~b}$ patients prior to treatment and had a negative impact on treatment outcomes, particularly in treatment-experienced genotype 1a patients. ${ }^{7}$ Nonetheless, all genotype 1a patients in this study were treatment naïve. Therefore, although RAS testing was not per-

\footnotetext{
Abbreviations:

APRI, aspartate aminotransferase to platelet ratio index; $\mathrm{CHC}$, chronic hepatitis C; DAAs, direct-acting antivirals; FIB-4, fibrosis-4; HCV, hepatitis C virus; LDV, ledipasvir; RAS, resistance-associated substitutions; SOF, sofosbuvir; SVR, sustained viral response
}

\section{Corresponding author: Moon Young Kim}

Division of Gastroenterology and Hepatology, Department of Internal Medicine, Yonsei University Wonju College of Medicine, 20 Ilsan-ro, Wonju 26426, Korea

Tel: +82-33-741-1229, Fax: +82-33-741-0951

E-mail:drkimmy@yonsei.ac.kr

https://orcid.org/0000-0002-2501-2206 
formed, the low SVR rate in genotype 1a was not considered to be due to RASs. Instead, it is presumed that the higher fibrosis scoring, including aspartate aminotransferase to platelet ratio index (APRI) and fibrosis-4 (FIB-4), in genotype 1a than in 1b reduced the treatment response. Moreover, high FIB-4 (>3.25) and APRI (>0.7) were inversely associated with end of treatment response and SVR12 achievement in this study, indicating that significant fibrosis is an alarming sign of poor treatment response. Nevertheless, these findings are not new and must be interpreted with caution for several reasons.

First, LDV/SOF use in advanced chronic kidney disease patients (estimated glomerular filtration rate $<30 \mathrm{~mL} / \mathrm{min} / 1.73 \mathrm{~m}^{2}$ ) might be of concern to some physicians because SOF and its metabolite GS-331007 are mainly eliminated through renal clearance. ${ }^{8}$ Unfortunately, as the author mentioned, renal function changes either during or end of the treatment were not analyzed. Although several studies have reported that SOF-based therapy is safe and effective in patients with severe chronic kidney disease, including hemodialysis patients, its safety has not yet been fully established. Hence, it is unfortunate that this largest real-life study did not provide information on whether SOF-based therapy affects renal function.

Next, recent the European Association for the Study of the Liver and American Association for the Study of Liver Diseases guidelines recommend pangenotypic DAA-based regimens (e.g., SOF/ velpatasvir and glecaprevir/pibrentasvir) for patients without cirrhosis or with compensated cirrhosis to improve access to HCV treatment and increase cure rate in any setting. ${ }^{4,9}$ Thus, wherever genotype determination is not available, simplified treatment without knowledge of the HCV genotype, and not affordable access to HCV care, is expected to improve HCV treatment. Given these latest trends, this study analyzing the effectiveness of LDV/ SOF may provide outdated results. However, pangenotype DAAbased therapy is not yet available in many countries, depending on the region and health system. SOF/velpatasvir and SOF/velpatasvir/voxilaprevir are not yet available in Korea. Thus, in countries with limited access to SOF/velpatasvir, LDV/SOF remains a good treatment option.

This study showed that the estimated mean values of non-invasive fibrosis markers, including APRI and FIB-4, decreased reliably both during and after treatment. A previous report showed that the improvement of fibrosis through liver stiffness measurement or non-invasive fibrosis serum markers predicted treatment response and prognosis in treated CHC patients. 10,11 Nonetheless, accurate diagnosis of fibrosis using tissue biopsy or liver stiffness measurement was limited. Therefore, whether this indicates a true fibrosis regression or merely the resolution of chronic liver inflammation with subsequent improvement of laboratory parameters remains unclear.

The World Health Organization has set ambitious goals for the control of viral hepatitis by $2030 .^{12}$ In the era of DAAs, where treatment efficacy and tolerability are no longer the major concerns, a comprehensive HCV care cascade is needed, including proper screening for unawareness of HCV, accurate diagnosis, and linking to medical care. ${ }^{13}$ Although the amount of clinical interest is low, the findings of this study conducted by the Mongolian government for Hepatitis Prevention, Control and Elimination are meaningful. Moreover, this large sized study showed the effectiveness of LDV/SOF at a fixed dose and treatment duration in helping to promote universal healthcare for $\mathrm{CHC}$ in countries with resource-limited settings.

\section{Authors' contribution}

SH Kang, manuscript writing and critical revision; MY Kim, critical revision and supervision

\section{Conflicts of Interest}

The authors have no conflicts to disclose.

\section{REFERENCES}

1. Polaris Observatory HCV Collaborators. Global prevalence and genotype distribution of hepatitis C virus infection in 2015: a modelling study. Lancet Gastroenterol Hepatol 2017;2:161-176.

2. Pimpin L, Cortez-Pinto H, Negro F, Corbould E, Lazarus JV, Webber $L$, et al. Burden of liver disease in Europe: epidemiology and analysis of risk factors to identify prevention policies. J Hepatol 2018;69:718 735.

3. European Association for the Study of the Liver. EASL Recommendations on Treatment of Hepatitis C 2018. J Hepatol 2018;69:461-511.

4. Ghany MG, Morgan TR; AASLD-IDSA Hepatitis C Guidance Panel. Hepatitis C guidance 2019 update: American Association for the Study of Liver Diseases-Infectious Diseases Society of America recommendations for testing, managing, and treating hepatitis $C$ virus infection. Hepatology 2020;71:686-721.

5. Asahina Y, Liu CJ, Gane E, Itoh Y, Kawada N, Ueno Y, et al. Twelve weeks of ledipasvir/sofosbuvir all-oral regimen for patients with chronic hepatitis C genotype 2 infection: integrated analysis of three clinical trials. Hepatol Res 2020;50:1109-1117.

6. Baatarkhuu O, Lee JS, Amarsanaa J, Kim DY, Ahn SH, Naranzul N, et 


\section{CLINICAL and MOLECULAR}

al. Efficacy and safety of ledipasvir/sofosbuvir in 5,028 Mongolian patients infected with genotype 1 hepatitis C virus: a multicenter study. Clin Mol Hepatol 2021;27:125-135.

7. Zeuzem S, Mizokami M, Pianko S, Mangia A, Han KH, Martin R, et al. NS5A resistance-associated substitutions in patients with genotype 1 hepatitis $C$ virus: prevalence and effect on treatment outcome. J Hepatol 2017;66:910-918.

8. U.S. Food and Drug Administration (FDA). HARVONI prescribing information. FDA web site, <https://www.accessdata.fda.gov/ drugsatfda_docs/label/2019/212477s000lbl.pdf>. Accessed 9 Nov 2020.

9. European Association for the Study of the Liver. EASL recommendations on treatment of hepatitis C: final update of the series 2 . J Hepatol 2020;73:1170-1218.
10. Moon C, Jung KS, Kim DY, Baatarkhuu O, Park JY, Kim BK, et al. Lower incidence of hepatocellular carcinoma and cirrhosis in hepatitis C patients with sustained virological response by pegylated interferon and ribavirin. Dig Dis Sci 2015;60:573-581.

11. Lee HW, Oh SR, Kim DY, Jeong Y, Kim S, Kim BK, et al. Daclatasvir plus asunaprevir for the treatment of patients with hepatitis $C$ virus genotype $1 \mathrm{~b}$ infection: real-world efficacy, changes in liver stiffness and fibrosis markers, and safety. Gut Liver 2018;12:324-330.

12. World Health Organization (WHO). Global health sector strategy on viral hepatitis 2016-2021. Towards ending viral hepatitis. Geneva: WHO Document Production Services, 2016.

13. Huang CF, Yu ML. Unmet needs of chronic hepatitis $C$ in the era of direct-acting antiviral therapy. Clin Mol Hepatol 2020;26:251-260. 